\title{
ESTILOS DIFERENCIALES DE PERSONALIDAD EN POBLACIÓN ADULTA ESPAÑOLA Y ARGENTINA
}

\author{
M. PILAR SÁNCHEZ-LÓPEZ ${ }^{1}$, MARÍA MARTINA² y MARTA E. APARICIO ${ }^{1}$ \\ ${ }^{1}$ Universidad Complutense de Madrid \\ ${ }^{2}$ Universidad de Buenos Aires ${ }^{1}$
}

(Recibido el 15 de junio de 1998)

\begin{abstract}
A partir de la administración del Inventario Millon de Estilos de Personalidad (MIPS) se han estudiado características de cada una de las 24 escalas que integran la prueba en sujetos españoles y argentinos para evaluar Metas Motivacionales, Estilos Cognitivos y Relaciones Interpersonales. Se calculó para cada una de las escalas la media aritmética y la desviación típica. Tomando como base estas medidas se analizan las diferencias entre medias calculando la prueba t de Student. Para el total de las muestras estudiadas se han encontrado diferencias estadísticamente significativas en dieciséis de esas escalas. Se analizan a su vez las diferencias según sexo en las dos poblaciones estudiadas. A partir de las escalas en las que se obtienen diferencias en cada una de las poblaciones se ha realizado un análisis discriminante para analizar las escalas del cuestionario que mejor permiten discriminar entre los sujetos. Se avanzan conclusiones relacionadas con la comparación transnacional de datos y su aplicación clínica.

Palabras clave: Estilos de Personalidad, Inventario MIPS, diferencias grupales, España, Argentina.
\end{abstract}

\section{Different personality styles in spanish and argentine adults samples}

Differences in Personality Styles based in data gathered administering MIPS (Millon Inventory of Personality Styles) are analyzed. The inventory was administered to two adults samples (Argentine, Spain). Based in Means and Standard Deviations scores for each of the 24 scales that integrate the technique Student't scores were obtained. We found significative statistical differences in sixteen of those scales for the total samples analyzed. Furthermore, the paper analyzes differences according to sex between the two populations. A discriminant analysis is carried out to study which scales of the questionnaire allow us to discriminate better between subjects. The conclusions include some considerations about the transnational data comparisons and their clinical application.

Key words: Personality Styles, MIPS inventory, groupal differences, Spain, Argentine.

\section{INTRODUCCIÓN}

En el presente trabajo comparamos los perfiles en población española y argentina obtenidos a partir del Inventario Millon de Estilos de Personalidad (MIPS). Este cuestionario ya ha sido validado en ambas poblaciones en un trabajo previo (Sánchez-López y Aparicio,

Correspondencia: M. Pilar Sánchez López. Facultad de Psicología, Campus de Somosaguas, s/n, 28223, Madrid. Teléfono: 913943 200. Fax: 913942820. Correo-e: psdif02@sis.ucm.es.
1998), cuyos resultados generales se presentan en el análisis del instrumento.

El modelo teórico de T. Millon es un modelo integrador interesante porque se concibe como una configuración de estrategias y tácticas, de las cuales se selecciona cada técnica de intervención no sólo por su eficacia en la resolución de ciertos rasgos patológicos, sino también por su contribución a la constelación general de procedimientos terapéuticos (Millon, Everly y Davis, 1995). Dado nuestro interés en el Estilo Psicológico 
como base del estudio de la diversidad humana (cfr., p.ej., Sánchez-López, 1997), el modelo de T. Millon es atractivo para nosotros porque el modelo que propone se concibe como «Estilos de Personalidad" e incluye como una de sus áreas los Estilos Cognitivos.

En la propuesta de Millon se obtienen los trastornos de personalidad enumerados en el Eje II del DSM, a partir de un conjunto de tres polaridades. Estas se basan en principios evolutivos básicos y en estrategias de supervivencia para proporcionar las hipótesis explicativas sobre las formas normales y clínicas de la personalidad. La primera de estas polaridades se basa en "objetivos de la existencia", es decir, en la propuesta de que existen dos tendencias opuestas en equilibrio, búsqueda del placer o evitación del dolor. La segunda se refiere a «estrategias de replicación", que implica dos elecciones en la búsqueda de refuerzo en el yo o en los otros. Y la tercera se relaciona con "modos de supervivencia primarios", es decir, modos de afrontamiento pasivos o activos. Se propone que a partir del conocimiento de los defectos o desequilibrios en estas tres polaridades es posible derivar de forma deductiva los trastornos de personalidad incluidos en una taxonomía creada por el propio Millon (Millon et al., 1995).

Dentro de este modelo teórico, Millon desarrolla diversos cuestionarios, de los cuales, el MIPS (Inventario Millon de Estilos de Personalidad) es un inventario que mide Estilos de Personalidad en la población general. El inventario está compuesto por 180 ítems de verdadero o falso que se distribuyen en 12 escalas bipolares y tres indicadores de validez: Impresión Positiva (tendencia de los sujetos a dar una imagen positiva de sí mismos), Impresión Negativa (tendencia a dar una imagen excesivamente negativa de sí mismos) y Consistencia. Las 12 escalas del MIPS se distribuyen en tres áreas: Estilos Cognitivos (EC, a partir de ahora), Metas Motivacionales (MM, a partir de ahora) y Relaciones Interpersonales (RI, a partir de ahora) (Millon, 1994).

En las MM se incluyen tres bipolaridades debido a las vinculaciones entre la teoría ecológica y la evolucionista. La fundamentación teórica de las MM se basa en los antecedentes conceptuales de estas ciencias, a través de tres formulaciones: existencia, adaptación y replicación. Estas tres polaridades se han venido desarrollando desde comienzos de siglo, en especial con las aportaciones de Freud, que propone tres polaridades: sujeto-objeto, placer-dolor y actividadpasividad. Millon elaboró estas polaridades, pero desde el ámbito del aprendizaje, lo que incluye tres dimensiones bipolares: refuerzo positivo vs. refuerzo negativo (placer-dolor), uno mismo vs. los demás como fuente del refuerzo y los estilos instrumentales activo vs. pasivo. A partir de este modelo teórico elabora las siguientes polaridades definitivas del cuestionario: Apertura-Preservación, Modificación-Acomodación e Individualismo-Protección. Las MM evalúan, por tanto, la orientación de la persona a obtener refuerzo del medio.

Las cuatro bipolaridades de los EC se basan en la perspectiva evolucionista y las contribuciones de Jung y Myers. Los EC se refieren a la forma en la que los organismos abordan su entorno. Pretenden evaluar los estilos o modos de procesamiento de la información. Jung propone una orientación cognitiva en una tipología que incluye tres polaridades: Extraversión/Introversión, Pensamiento/Sentimiento y Sensación/Intuición. Millon elabora un modelo que agrupa las actividades cognitivas teniendo en cuenta dos funciones superiores. Una se relaciona con el origen de los datos recogidos, es decir, con "las fuentes de información"; y otra con los métodos utilizados por el individuo para reconstruir 
esos datos, "los procesos de transformación». Cada una de estas funciones fue dividida a su vez en dos polaridades: las fuentes de información se dividieron en externas vs. internas y tangibles vs. intangibles. Los procesos de transformación fueron divididos en intelectivos vs. afectivos y asimilativos vs. imaginativos. Las polaridades definitivas son: ExtravertidoIntravertido, Sensitivo-Intuitivo, Reflexivo-Afectivo, y Sistematizador-Innovador.

Por último, las cinco bipolaridades de las RI están relacionadas con las contribuciones de Sullivan y Leary y el modelo de los Cinco Grandes Factores. Tanto el enfoque interpersonal de Leary, basado en el de Sullivan, como la fundamentación de los Cinco Grandes factores en términos descriptivos comunes de la interacción humana, están orientados hacia los rasgos conductuales, es decir, hacia los rasgos que caracterizan las acciones de la gente, lo que la gente hace y no lo que la motiva o el modo como funcionan cognitivamente; pero ninguno de los modelos anteriores ha introducido un componente interpersonal, el cual Millon se propone abordar con el fin de evaluar el estilo de relacionarse con los demás. Los cinco pares se basan en una teoría biosocial/de aprendizaje basada en la naturaleza y la fuente de los reforzadores y en las estrategias instrumentales activas y pasivas. Las polaridades que incluye son: Retraimiento-Comunicatividad, Duda-Seguridad, Discrepancia-Conformidad, Sometimiento-Control e Insatisfacción-Concordancia.

Por tanto, el objetivo del estudio es analizar las diferencias en las escalas del MIPS entre dos poblaciones diferentes, un grupo de personas argentinas y otro de españoles. Además se analizarán las escalas del MIPS que mejor nos permiten diferenciar entre los dos grupos de sujetos. Se trata de comparar grupos de sujetos pertenecientes a dos subculturas de una macrocultura global, la occidental.
Nos centramos en dos grandes ciudades, una europea (Madrid), la otra sudamericana (Buenos Aires), que comparten además, idioma común. El cuestionario ha sido adaptado a las variedades idiomáticas de cada uno de los países, España y Argentina.

\section{MÉTODO}

\section{Participantes}

En este trabajo han participado sujetos de dos países: España y Argentina. El grupo español está compuesto por 624 sujetos, de los cuales $428(68,6 \%)$ son mujeres y $195(31,3 \%)$ hombres, con una edad media de 27,59 años y una desviación típica de 9,603 (mínimo de 17 y máximo de 78 años).

El grupo argentino está compuesto por 449 sujetos, de los cuales 299 son mujeres $(66,6 \%)$ y 150 hombres $(33,4 \%)$. La media de edad es de 27 años y una desviación típica de 11,9 (mínimo de 15 y máximo de 99 años).

\section{Instrumento}

Se ha realizado la adaptación del original inglés (Millon, 1994) en primer lugar en su traducción Argentina y después en la adaptación realizada en España (Sánchez-López y Aparicio, 1998). En la ciudad de Buenos Aires se ha empleado la versión del Inventario que ha publicado la editorial Paidós, edición que fue diseñada con el asesoramiento de una de las autoras (M.M. Casullo). Se tradujo la versión original del inglés al español, luego esa versión fue pasada nuevamente al inglés (back translation) por dos psicólogos bilingües. Sobre la base de esta última versión, se realizó una prueba piloto $(\mathrm{N}=25)$. Se les solicitó a estos sujetos que leyeran los diferentes items que 
integran el Inventario y que señalaran aquellos cuya comprensión les resultaba difícil o confusa. Estos datos fueron tenidos en cuenta en la redacción última de la prueba que editó Paidós (Millon,1994).

En la ciudad de Madrid se ha aplicado la versión del Inventario realizada (Aparicio y Sánchez-López, en prensa) adaptada a la variedad idiomática del español que se habla en España. Con la versión argentina, y siempre teniendo como referencia el original estadounidense, se realizó un estudio idiomático del cuestionario mediante su aplicación a un grupo de sujetos a los que se les pedía que anotasen los ítems que les resultaban poco familiares o inadecuados a nuestro uso lingüístico. A continuación, se aplicó el cuestionario a un grupo reducido de sujetos $(\mathrm{N}=20)$, a los que se les pedía su opinión y sus comentarios sobre la comprensión del español utilizado y la adecuación a nuestra variedad idiomática. A continuación se modificaron aquellos ítems que al menos el $60 \%$ de los sujetos preguntados habían considerado inadecuados. El siguiente paso fue aplicar de nuevo el cuestionario, repitiendo el proceso hasta que los sujetos no encontraban ninguna dificultad para comprender el cuestionario.

En estudios previos se ha mostrado la fiabilidad del instrumento $\{0,77$ en EEUU, Millon, 1994; 0,72 en España, Aparicio y Sánchez-López, en prensa; 0,70 en Argentina, Sánchez-López y Aparicio, 1998). El análisis de la validez y de las correlaciones en las escalas aporta resultados similares, al menos para los grupos estadounidenses y españoles (Aparicio y Sánchez-López, en prensa). Las escalas del MIPS se superponen e intercorrelacionan de diversos modos; en general se desprende un patrón de relaciones convergentes y divergentes entre las escalas que concuerda ampliamente con las expectativas basadas en la naturaleza de los constructos que se miden.
Por otro lado, se confirma la bipolaridad (A y B) de las escalas propuesta por Millon. En general, cuando los polos A y $B$ de una misma escala saturan juntos en el mismo factor, siempre lo hacen con signos opuestos. Además, existen algunos polos (A y B) que saturan siempre juntos en el mismo factor y nunca aisladamente (A o B) en otro. Se confirma, por lo tanto, a partir de la matriz de correlaciones y del análisis factorial que la estructura del MIPS en la población española es semejante al modelo propuesto por Millon.

\section{Procedimiento}

Los sujetos contestaban al cuestionario, que se les repartía con unas normas por escrito. En la muestra española la aplicación se realizaba en salones de clase en grupos de aproximadamente 50 sujetos cada vez. En Argentina la aplicación se concretó de forma individualizada. A todos los participantes se le aseguraba el anonimato de sus resultados y se les pedía que fueran sinceros en sus respuestas.

\section{Análisis de datos}

Para las diferencias de medias entre ambos grupos usamos la prueba $t$ de Student. A partir de estos datos se ha realizado un análisis discriminante para analizar las escalas que más diferencian entre los sujetos. Ambas técnicas se han realizado a través del paquete estadístico SPSS (v. 6.1.2.).

\section{RESULTADOS}

En la Tabla 1 se presentan los resultados de las escalas del MIPS comparando los datos del grupo de personas españolas y del grupo de argentinos. En esta tabla 
observamos que existen diferencias en 13 escalas del MIPS y en la escala de Impresion Positiva (los argentinos tienen mayor tendencia que los españoles a dar una imagen positiva de sí mismos, al menos en los grupos que hemos analizado).

Para analizar qué escalas nos permiten diferenciar mejor entre los españoles y argentinos de nuestro estudio realizamos un análisis discriminante con aquellas escalas en las que se habían obtenido diferencias significativas, es decir, las 13 escalas que comentábamos anteriormente. Los resultados de la función discriminante canónica fueron como sigue: valor propio $=0,09$, varianza $=100 \%$, correlación canónica $=0,28$, lambda de Wilks $=0,92, y$ $\chi^{2}(13)=72,00, p<0,0001$; los coeficientes de estructura se presentan en la Tabla 2.

Tabla 2. Coeficientes de estructura de las escalas significativas en los datos de comparación entre personas españolas y argentinas

\begin{tabular}{llr}
\hline & & Función 1 \\
\hline S12A. & Insatisfacción. & $\mathbf{- 0 , 5 4 7 3 5}$ \\
S3B. & Protección. & $\mathbf{0 , 5 0 3 1 9}$ \\
S10A. & Discrepancia. & $-\mathbf{0 , 4 2 1 0 2}$ \\
S9B. & Firmeza. & $\mathbf{0 , 4 1 7 2 1}$ \\
S6B. & Afectividad. & $\mathbf{0 , 4 1 5 4 4}$ \\
S10B. & Conformismo. & $\mathbf{0 , 3 3 1 5 7}$ \\
S1A. & Apertura. & $\mathbf{0 , 3 0 6 1 1}$ \\
S11A. & Sometimiento. & $-\mathbf{0 , 2 7 0 6 0}$ \\
S12B. & Concordancia. & $\mathbf{0 , 2 5 9 7 9}$ \\
S6A. & Reflexión. & $-\mathbf{0 , 2 5 8 7 6}$ \\
S2A. & Modificación. & $\mathbf{0 , 2 2 8 8 2}$ \\
S2B. & Acomodación. & $-0,20368$ \\
S9A. & Vacilación. & $-0,17252$ \\
\hline
\end{tabular}

Tabla 1. Medias y desviaciones típicas de las escalas del MIPS en los datos totales españoles y argentinos

\begin{tabular}{|c|c|c|c|c|c|c|}
\hline \multirow{2}{*}{$\begin{array}{c}\text { Totales } \\
\text { Escalas MIPS }\end{array}$} & \multicolumn{2}{|c|}{ España } & \multicolumn{2}{|c|}{ Argentina } & \multirow[b]{2}{*}{$\mathrm{T}$} & \multirow[b]{2}{*}{ N. Sig. } \\
\hline & Media & DT & Media & DT & & \\
\hline $\begin{array}{l}\text { 1A. APERTURA } \\
\text { 1B. PRESERVACIÓN }\end{array}$ & $\begin{array}{l}23,87 \\
16,05\end{array}$ & $\begin{array}{r}7,51 \\
10,07\end{array}$ & $\begin{array}{l}25,5 \\
14,9\end{array}$ & $\begin{array}{l}6,9 \\
9,7\end{array}$ & $\begin{array}{r}-3,54 \\
1,78\end{array}$ & $\begin{array}{l}0,000^{\star} \\
0,076\end{array}$ \\
\hline $\begin{array}{l}\text { 2A. MODIFICACIÓN } \\
\text { 2B. ACOMODACIÓN }\end{array}$ & $\begin{array}{l}34,01 \\
19,01\end{array}$ & $\begin{array}{l}4,11 \\
9,85\end{array}$ & $\begin{array}{l}34,8 \\
27,5\end{array}$ & $\begin{array}{l}3,8 \\
9,1\end{array}$ & $\begin{array}{r}-3,17 \\
2,47\end{array}$ & $\begin{array}{l}0,002^{\star} \\
0,014^{*}\end{array}$ \\
\hline $\begin{array}{l}\text { 3A. INDIVIDUALISMO } \\
\text { 3B. PROTECCIÓN }\end{array}$ & $\begin{array}{l}19,06 \\
29,27\end{array}$ & $\begin{array}{l}8,16 \\
8,25\end{array}$ & $\begin{array}{l}18,9 \\
32,0\end{array}$ & $\begin{array}{l}7,2 \\
7,6\end{array}$ & $\begin{array}{r}0,43 \\
-5,44\end{array}$ & $\begin{array}{l}0,667 \\
0,000^{*}\end{array}$ \\
\hline $\begin{array}{l}\text { 4A. EXTRAVERSIÓN } \\
\text { 4B. INTROVERSIÓN }\end{array}$ & $\begin{array}{l}27,51 \\
10,28\end{array}$ & $\begin{array}{l}8,56 \\
7,23\end{array}$ & $\begin{array}{r}28,1 \\
9,7\end{array}$ & $\begin{array}{l}8,1 \\
7,0\end{array}$ & $\begin{array}{l}-1,25 \\
1,2\end{array}$ & $\begin{array}{l}0,213 \\
0,229\end{array}$ \\
\hline $\begin{array}{l}\text { 5A. SENSACIÓN } \\
\text { 5B. INTUICIÓN }\end{array}$ & $\begin{array}{l}18,15 \\
22\end{array}$ & $\begin{array}{l}6,3 \\
8,45\end{array}$ & $\begin{array}{l}17,7 \\
22,3\end{array}$ & $\begin{array}{l}6,4 \\
8,1\end{array}$ & $\begin{array}{l}1,38 \\
-0,7\end{array}$ & $\begin{array}{l}0,167 \\
0,484\end{array}$ \\
\hline $\begin{array}{l}\text { 6A. REFLEXIÓN } \\
\text { 6B. AFECTIVIDAD }\end{array}$ & $\begin{array}{l}14,94 \\
28,49\end{array}$ & $\begin{array}{l}8,09 \\
8,88\end{array}$ & $\begin{array}{l}23,7 \\
30,4\end{array}$ & $\begin{array}{l}7,1 \\
7,9\end{array}$ & $\begin{array}{r}2,79 \\
-3,71\end{array}$ & $\begin{array}{l}0,005^{\star} \\
0,000^{\star}\end{array}$ \\
\hline $\begin{array}{l}\text { 7A. SISTEMATIZACIÓN } \\
\text { 7B. INNOVACIÓN }\end{array}$ & $\begin{array}{l}35,62 \\
27,29\end{array}$ & $\begin{array}{r}11,18 \\
8,89\end{array}$ & $\begin{array}{l}37,0 \\
27,3\end{array}$ & $\begin{array}{r}11,1 \\
8,5\end{array}$ & $\begin{array}{l}-1,84 \\
-0,31\end{array}$ & $\begin{array}{l}0,065 \\
0,759\end{array}$ \\
\hline $\begin{array}{l}\text { 8A. RETRAIMIENTO } \\
\text { 8B. COMUNICATIVID. }\end{array}$ & $\begin{array}{l}16,79 \\
33,52\end{array}$ & $\begin{array}{r}9,02 \\
11,05\end{array}$ & $\begin{array}{l}16,4 \\
34,6\end{array}$ & $\begin{array}{r}9,0 \\
10,4\end{array}$ & $\begin{array}{r}0,71 \\
-1,64\end{array}$ & $\begin{array}{l}0,476 \\
0,100\end{array}$ \\
\hline $\begin{array}{l}\text { 9A. VACILACIÓN } \\
\text { 9B. FIRMEZA }\end{array}$ & $\begin{array}{l}16,82 \\
33,2\end{array}$ & $\begin{array}{l}10,69 \\
11,11\end{array}$ & $\begin{array}{l}15,4 \\
35,9\end{array}$ & $\begin{array}{l}9,9 \\
9,9\end{array}$ & $\begin{array}{r}2,12 \\
-4,08\end{array}$ & $\begin{array}{l}0,034^{*} \\
0,000^{*}\end{array}$ \\
\hline $\begin{array}{l}\text { 10A. DISCREPANCIA } \\
\text { 10B. CONFORMISMO }\end{array}$ & $\begin{array}{l}21,48 \\
16,75\end{array}$ & $\begin{array}{l}8,21 \\
4,4\end{array}$ & $\begin{array}{l}19,2 \\
17,7\end{array}$ & $\begin{array}{l}8,1 \\
4,6\end{array}$ & $\begin{array}{r}4,18 \\
-3,42\end{array}$ & $\begin{array}{l}0,000^{*} \\
0,001^{*}\end{array}$ \\
\hline $\begin{array}{l}\text { 11A. SOMETIMIENTO } \\
\text { 11B. CONTROL }\end{array}$ & $\begin{array}{l}15,8 \\
24,97\end{array}$ & $\begin{array}{l}8,19 \\
7,64\end{array}$ & $\begin{array}{l}14,4 \\
25,3\end{array}$ & $\begin{array}{l}8,4 \\
6,8\end{array}$ & $\begin{array}{r}2,66 \\
-0,76\end{array}$ & $\begin{array}{l}0,008^{\star} \\
0,448\end{array}$ \\
\hline $\begin{array}{l}\text { 12A. INSATISFACCION } \\
\text { 12B. CONCORDANCIA }\end{array}$ & $\begin{array}{l}24,37 \\
31,96\end{array}$ & $\begin{array}{l}8,67 \\
8,71\end{array}$ & $\begin{array}{l}21,0 \\
33,3\end{array}$ & $\begin{array}{l}9,4 \\
7,6\end{array}$ & $\begin{array}{r}5,61 \\
-2,49\end{array}$ & $\begin{array}{l}0,000^{*} \\
0,013^{*}\end{array}$ \\
\hline $\begin{array}{l}\text { IMPRES. NEGATIVA } \\
\text { IMPRES. POSITIVA }\end{array}$ & $\begin{array}{l}3,08 \\
3,55\end{array}$ & $\begin{array}{l}2,38 \\
1,99\end{array}$ & $\begin{array}{l}3,2 \\
4,3\end{array}$ & $\begin{array}{l}2,2 \\
2,2\end{array}$ & $\begin{array}{l}-0,73 \\
-5,33\end{array}$ & $\begin{array}{l}0,468 \\
0,000^{\star}\end{array}$ \\
\hline
\end{tabular}

Nota: ${ }^{*} p<0,05$ 
En la Tabla 3 analizamos las diferencias existentes en las mujeres de ambos grupos (españolas y argentinas) observando que existen diferencias en $10 \mathrm{de}$ las 24 escalas.

En la Tabla 4 se presenta la comparación entre hombres argentinos y españoles, de la cual extraemos que existen diferencias en 9 escalas del MIPS y en el índice de control de Impresión Positiva, en la que se observa que son los hombres argentinos los que tienen mayor tendencia a dar una imagen positiva de sí mismos.

Con objeto de analizar las diferencias entre sexo en el grupo de personas espa- ñolas hemos realizado una comparación en la Tabla 5 y se obtienen diferencias en 16 escalas y en la escala de Impresión Negativa, en la que observamos que las mujeres tienden a dar una imagen más negativa de sí mismas que los hombres.

Al igual que hicimos con los datos comparativos entre españoles y argentinos, ahora analizamos las variables que mejor discriminan entre hombres y mujeres españoles mediante un análisis discriminante. Los resultados de la función discriminante canónica fueron: valor propio $=0,21$, varianza $=100 \%$, correlación canónica $=0,42$, lambda de Wilks $=0,82$, y $\chi^{2}(4)=109,87, p<0,0001$; los coefi-

Tabla 3. Medias y Desviaciones Estándar de las escalas del MIPS en los datos del grupo de mujeres españolas y argentinas

\begin{tabular}{|c|c|c|c|c|c|c|}
\hline Totales & \multicolumn{2}{|c|}{ España } & \multicolumn{2}{|c|}{ Argentina } & \multirow[b]{2}{*}{$\mathrm{T}$} & \multirow[b]{2}{*}{ N. Sig. } \\
\hline Escalas MIPS & Media & DT & Media & DT & & \\
\hline $\begin{array}{l}\text { 1A. APERTURA } \\
\text { 1B. PRESERVACION }\end{array}$ & $\begin{array}{l}23,1 \\
17,53\end{array}$ & $\begin{array}{c}7,48 \\
10,1\end{array}$ & $\begin{array}{l}25,2 \\
15,6\end{array}$ & $\begin{array}{l}7,1 \\
9,9\end{array}$ & $\begin{array}{r}-3,76 \\
2,56\end{array}$ & $\begin{array}{l}0,000^{\star} \\
0,011^{*}\end{array}$ \\
\hline $\begin{array}{l}\text { 2A. MODIFICACIÓN } \\
\text { 2B. ACOMODACIÓN }\end{array}$ & $\begin{array}{l}33,84 \\
20,01\end{array}$ & $\begin{array}{l}4,18 \\
9,73\end{array}$ & $\begin{array}{l}14,5 \\
18,2\end{array}$ & $\begin{array}{l}3,9 \\
9,0\end{array}$ & $\begin{array}{r}-1,83 \\
2,49\end{array}$ & $\begin{array}{l}0,068 \\
0,013^{*}\end{array}$ \\
\hline $\begin{array}{l}\text { 3A. INDIVIDUALISMO } \\
\text { 3B. PROTECCIÓN }\end{array}$ & $\begin{array}{l}17,52 \\
30,96\end{array}$ & $\begin{array}{l}7,72 \\
7,55\end{array}$ & $\begin{array}{l}18,1 \\
32,6\end{array}$ & $\begin{array}{l}7,3 \\
7,5\end{array}$ & $\begin{array}{l}-0,94 \\
-2,81\end{array}$ & $\begin{array}{l}0,347 \\
0,005^{*}\end{array}$ \\
\hline $\begin{array}{l}\text { 4A. EXTRAVERSIÓN } \\
\text { 4B. INTROVERSIÓN }\end{array}$ & $\begin{array}{l}27,92 \\
10,05\end{array}$ & $\begin{array}{l}8,19 \\
7,26\end{array}$ & $\begin{array}{r}28,1 \\
9,3\end{array}$ & $\begin{array}{l}8,2 \\
6,9\end{array}$ & $\begin{array}{r}-0,31 \\
1,37\end{array}$ & $\begin{array}{l}0,756 \\
0,170\end{array}$ \\
\hline $\begin{array}{l}\text { 5A. SENSACIÓN } \\
\text { 5B. INTUICIÓN }\end{array}$ & $\begin{array}{l}17,92 \\
22,38\end{array}$ & $\begin{array}{l}6,43 \\
8,58\end{array}$ & $\begin{array}{l}17,2 \\
23,2\end{array}$ & $\begin{array}{l}6,5 \\
7,9\end{array}$ & $\begin{array}{r}1,51 \\
-1,26\end{array}$ & $\begin{array}{l}0,131 \\
0,208\end{array}$ \\
\hline $\begin{array}{l}\text { 6A. REFLEXIÓN } \\
\text { 6B. AFECTIVIDAD }\end{array}$ & $\begin{array}{l}13,34 \\
20,26\end{array}$ & $\begin{array}{l}7,6 \\
8,25\end{array}$ & $\begin{array}{l}12,4 \\
31,4\end{array}$ & $\begin{array}{l}6,9 \\
7,7\end{array}$ & $\begin{array}{l}1,65 \\
-1,8\end{array}$ & $\begin{array}{l}0,100 \\
0,072\end{array}$ \\
\hline $\begin{array}{l}\text { 7A. SISTEMATIZACIÓN } \\
\text { 7B. INNOVACIÓN }\end{array}$ & $\begin{array}{l}35,01 \\
27,35\end{array}$ & $\begin{array}{r}11,04 \\
8,86\end{array}$ & $\begin{array}{l}36,2 \\
27,7\end{array}$ & $\begin{array}{r}11,1 \\
8,3\end{array}$ & $\begin{array}{l}-1,24 \\
-0,56\end{array}$ & $\begin{array}{l}0,216 \\
0,575\end{array}$ \\
\hline $\begin{array}{l}\text { 8A. RETRAIMIENTO } \\
\text { 8B. COMUNICATIVID. }\end{array}$ & $\begin{array}{l}16,19 \\
32,84\end{array}$ & $\begin{array}{r}8,68 \\
10,59\end{array}$ & $\begin{array}{l}16,4 \\
33,9\end{array}$ & $\begin{array}{r}9,3 \\
10,3\end{array}$ & $\begin{array}{l}-0,23 \\
-1,27\end{array}$ & $\begin{array}{l}0,821 \\
0,204\end{array}$ \\
\hline $\begin{array}{l}\text { 9A. VACILACIÓN } \\
\text { 9B. FIRMEZA }\end{array}$ & $\begin{array}{l}17,76 \\
31,65\end{array}$ & $\begin{array}{l}10,7 \\
10,63\end{array}$ & $\begin{array}{l}15,9 \\
35,2\end{array}$ & $\begin{array}{r}10,3 \\
9,9\end{array}$ & $\begin{array}{r}2,22 \\
-4,37\end{array}$ & $\begin{array}{l}0,027^{*} \\
0,000^{*}\end{array}$ \\
\hline $\begin{array}{l}\text { 10A. DISCREPANCIA } \\
\text { 10B. CONFORMISMO }\end{array}$ & $\begin{array}{l}20,84 \\
16,88\end{array}$ & $\begin{array}{l}7,89 \\
4,2\end{array}$ & $\begin{array}{l}19,3 \\
17,7\end{array}$ & $\begin{array}{l}8,3 \\
4,3\end{array}$ & $\begin{array}{r}2,53 \\
-2,43\end{array}$ & $\begin{array}{l}0,012^{*} \\
0,015^{*}\end{array}$ \\
\hline $\begin{array}{l}\text { 11A. SOMETIMIENTO } \\
\text { 11B. CONTROL }\end{array}$ & $\begin{array}{l}16,71 \\
23,56\end{array}$ & $\begin{array}{l}8,18 \\
7,02\end{array}$ & $\begin{array}{l}14,9 \\
24,7\end{array}$ & $\begin{array}{l}8,7 \\
6,7\end{array}$ & $\begin{array}{r}2,72 \\
-2,09\end{array}$ & $\begin{array}{l}0,007^{*} \\
0,037\end{array}$ \\
\hline $\begin{array}{l}\text { 12A. INSATISFACCIÓN } \\
\text { 12B. CONCORDANCIA }\end{array}$ & $\begin{array}{l}24,55 \\
33,8\end{array}$ & $\begin{array}{l}8,69 \\
8,14\end{array}$ & $\begin{array}{l}20,7 \\
34,2\end{array}$ & $\begin{array}{l}9,7 \\
7,3\end{array}$ & $\begin{array}{r}5,15 \\
-0,69\end{array}$ & $\begin{array}{l}0,000^{*} \\
0,492\end{array}$ \\
\hline $\begin{array}{l}\text { IMPRES.NEGATIVA } \\
\text { IMPRES.POSITIVA }\end{array}$ & $\begin{array}{l}3,31 \\
3,62\end{array}$ & $\begin{array}{l}2,42 \\
2,02\end{array}$ & $\begin{array}{l}3,3 \\
4,2\end{array}$ & $\begin{array}{l}2,3 \\
2,0\end{array}$ & $\begin{array}{l}-0,07 \\
-3,22\end{array}$ & $\begin{array}{l}0,945 \\
0,165\end{array}$ \\
\hline
\end{tabular}

Nota: * $\mathrm{p}<0,05$ 
Tabla 4. Medias y Desviaciones Estándar de las escalas del MIPS en los datos del grupo de hombres españoles y argentinos

\begin{tabular}{|c|c|c|c|c|c|c|}
\hline \multirow{2}{*}{$\frac{\text { Totales }}{\text { Escalas MIPS }}$} & \multicolumn{2}{|c|}{ España } & \multicolumn{2}{|c|}{ Argentina } & \multirow[b]{2}{*}{$\mathrm{T}$} & \multirow[b]{2}{*}{ N. Sig. } \\
\hline & Media & $\mathrm{DT}$ & Media & DT & & \\
\hline $\begin{array}{l}\text { 1A. APERTURA } \\
\text { 1B. PRESERVACIÓN }\end{array}$ & $\begin{array}{l}25,5 \\
12,87\end{array}$ & $\begin{array}{l}7,32 \\
9,25\end{array}$ & $\begin{array}{l}26,2 \\
13,5\end{array}$ & $\begin{array}{l}6,5 \\
9,1\end{array}$ & $\begin{array}{l}-0,72 \\
-0,83\end{array}$ & $\begin{array}{l}0,471 \\
0,408\end{array}$ \\
\hline $\begin{array}{l}\text { 2A. MODIFICACIÓN } \\
\text { 2B. ACOMODACIÓN }\end{array}$ & $\begin{array}{l}34,37 \\
16,9\end{array}$ & $\begin{array}{l}3,94 \\
9,76\end{array}$ & $\begin{array}{l}35,6 \\
16,2\end{array}$ & $\begin{array}{l}3,7 \\
9,1\end{array}$ & $\begin{array}{r}-2,93 \\
0,67\end{array}$ & $\begin{array}{l}0,004^{*} \\
0,501\end{array}$ \\
\hline $\begin{array}{l}\text { 3A. INDIVIDUALISMO } \\
\text { 3B. PROTECCIÓN }\end{array}$ & $\begin{array}{l}22,45 \\
25,63\end{array}$ & $\begin{array}{l}8,11 \\
8,54\end{array}$ & $\begin{array}{l}20,4 \\
30,7\end{array}$ & $\begin{array}{l}6,9 \\
7,7\end{array}$ & $\begin{array}{r}2,52 \\
-5,73\end{array}$ & $\begin{array}{l}0,012^{\star} \\
0,000^{\star}\end{array}$ \\
\hline $\begin{array}{l}\text { 4A. EXTRAVERSIÓN } \\
\text { 4B. INTROVERSIÓN }\end{array}$ & $\begin{array}{l}26,55 \\
10,81\end{array}$ & $\begin{array}{l}9,26 \\
7,17\end{array}$ & $\begin{array}{l}28,2 \\
10,6\end{array}$ & $\begin{array}{l}8,0 \\
7,2\end{array}$ & $\begin{array}{r}-1,81 \\
0,21\end{array}$ & $\begin{array}{l}0,072 \\
0,834\end{array}$ \\
\hline $\begin{array}{l}\text { 5A. SENSACIÓN } \\
\text { 5B. INTUICIÓN }\end{array}$ & $\begin{array}{l}18,64 \\
21,24\end{array}$ & $\begin{array}{l}6,01 \\
8,1\end{array}$ & $\begin{array}{l}18,6 \\
20,5\end{array}$ & $\begin{array}{l}6,2 \\
8,1\end{array}$ & $\begin{array}{l}0,33 \\
0,49\end{array}$ & $\begin{array}{l}0,744 \\
0,622\end{array}$ \\
\hline $\begin{array}{l}\text { 6A. REFLEXIÓN } \\
\text { 6B. AFECTIVIDAD }\end{array}$ & $\begin{array}{l}18,39 \\
24,69\end{array}$ & $\begin{array}{l}8,05 \\
8,99\end{array}$ & $\begin{array}{l}16,2 \\
28,6\end{array}$ & $\begin{array}{l}6,8 \\
7,9\end{array}$ & $\begin{array}{r}2,89 \\
-4,22\end{array}$ & $\begin{array}{l}0,004^{*} \\
0,000^{*}\end{array}$ \\
\hline $\begin{array}{l}\text { 7A. SISTEMATIZACIÓN } \\
\text { 7B. INNOVACIÓN }\end{array}$ & $\begin{array}{l}36,91 \\
27,16\end{array}$ & $\begin{array}{c}11,41 \\
9\end{array}$ & $\begin{array}{l}38,7 \\
26,6\end{array}$ & $\begin{array}{r}11,2 \\
8,9\end{array}$ & $\begin{array}{r}-1,34 \\
0,22\end{array}$ & $\begin{array}{l}0,183 \\
0,823\end{array}$ \\
\hline $\begin{array}{l}\text { 8A. RETRAIMIENTO } \\
\text { 8B. COMUNICATIVID. }\end{array}$ & $\begin{array}{l}18,15 \\
34,95\end{array}$ & $\begin{array}{l}9,56 \\
11,9\end{array}$ & $\begin{array}{l}16,5 \\
36,2\end{array}$ & $\begin{array}{l}8,5 \\
9,7\end{array}$ & $\begin{array}{c}1,7 \\
-1,09\end{array}$ & $\begin{array}{l}0,089 \\
0,275\end{array}$ \\
\hline $\begin{array}{l}\text { 9A. VACILACIÓN } \\
\text { 9B. FIRMEZA }\end{array}$ & $\begin{array}{l}14,84 \\
36,48\end{array}$ & $\begin{array}{l}10,43 \\
11,42\end{array}$ & $\begin{array}{l}14,3 \\
37,5\end{array}$ & $\begin{array}{l}9,0 \\
9,8\end{array}$ & $\begin{array}{r}0,44 \\
-0,89\end{array}$ & $\begin{array}{l}0,664 \\
0,372\end{array}$ \\
\hline $\begin{array}{l}\text { 10A. DISCREPANCIA } \\
\text { 10B. CONFORMISMO }\end{array}$ & $\begin{array}{l}22,9 \\
16,45\end{array}$ & $\begin{array}{l}8,73 \\
4,81\end{array}$ & $\begin{array}{l}19,2 \\
17,8\end{array}$ & $\begin{array}{l}7,6 \\
5,2\end{array}$ & $\begin{array}{r}3,88 \\
-2,47\end{array}$ & $\begin{array}{l}0,000^{*} \\
0,014^{*}\end{array}$ \\
\hline $\begin{array}{l}\text { 11A. SOMETIMIENTO } \\
\text { 11B. CONTROL }\end{array}$ & $\begin{array}{l}13,88 \\
27,98\end{array}$ & $\begin{array}{l}7,88 \\
8,07\end{array}$ & $\begin{array}{l}13,2 \\
26,5\end{array}$ & $\begin{array}{l}7,5 \\
6,8\end{array}$ & $\begin{array}{l}0,65 \\
1,79\end{array}$ & $\begin{array}{l}0,519 \\
0,075\end{array}$ \\
\hline $\begin{array}{l}\text { 12A. INSATISFACCIÓN } \\
\text { 12B. CONCORDANCIA }\end{array}$ & $\begin{array}{l}24,03 \\
27,93\end{array}$ & $\begin{array}{l}8,65 \\
8,61\end{array}$ & $\begin{array}{l}21,7 \\
31,3\end{array}$ & $\begin{array}{l}8,7 \\
7,8\end{array}$ & $\begin{array}{r}2,22 \\
-3,53\end{array}$ & $\begin{array}{l}0,027^{\star} \\
0,000^{\star}\end{array}$ \\
\hline $\begin{array}{l}\text { IMPRES. NEGATIVA } \\
\text { IMPRES. POSITIVA }\end{array}$ & $\begin{array}{l}2,58 \\
3,38\end{array}$ & $\begin{array}{l}2,2 \\
1,93\end{array}$ & $\begin{array}{l}2,9 \\
4,6\end{array}$ & $\begin{array}{l}2,2 \\
2,6\end{array}$ & $\begin{array}{l}-1,3 \\
-4,51\end{array}$ & $\begin{array}{l}0,194 \\
0,000^{\star}\end{array}$ \\
\hline
\end{tabular}

Nota: * $\mathrm{p}<0,05$

Tabla 5. Diferencias entre sexo en el grupo de españoles

\begin{tabular}{|c|c|c|c|c|c|c|}
\hline Totales & \multicolumn{2}{|c|}{ España } & \multicolumn{2}{|c|}{ Argentina } & \multirow[b]{2}{*}{$\mathrm{T}$} & \multirow[b]{2}{*}{ N. Sig. } \\
\hline Escalas MIPS & Media & DT & Media & DT & & \\
\hline $\begin{array}{l}\text { 1A. APERTURA } \\
\text { 1B. PRESERVACIÓN }\end{array}$ & $\begin{array}{l}23,1 \\
17,53\end{array}$ & $\begin{array}{l}7,48 \\
10,1\end{array}$ & $\begin{array}{l}25,5 \\
12,87\end{array}$ & $\begin{array}{l}7,32 \\
9,25\end{array}$ & $\begin{array}{r}-3,73 \\
5,65\end{array}$ & $\begin{array}{l}0,000^{*} \\
0,000^{*}\end{array}$ \\
\hline $\begin{array}{l}\text { 2A. MODIFICACIÓN } \\
\text { 2B. ACOMODACIÓN }\end{array}$ & $\begin{array}{l}33,84 \\
20,01\end{array}$ & $\begin{array}{l}4,18 \\
9,73\end{array}$ & $\begin{array}{l}34,37 \\
16,9\end{array}$ & $\begin{array}{l}3,94 \\
9,76\end{array}$ & $\begin{array}{r}-1,49 \\
3,68\end{array}$ & $\begin{array}{l}0,137 \\
0,000^{*}\end{array}$ \\
\hline $\begin{array}{l}\text { 3A. INDIVIDUALISMO } \\
\text { 3B. PROTECCIÓN }\end{array}$ & $\begin{array}{l}17,52 \\
30,96\end{array}$ & $\begin{array}{l}7,72 \\
7,55\end{array}$ & $\begin{array}{l}22,45 \\
25,63\end{array}$ & $\begin{array}{l}8,11 \\
8,54\end{array}$ & $\begin{array}{r}-7,23 \\
7,82\end{array}$ & $\begin{array}{l}0,000^{*} \\
0,000^{*}\end{array}$ \\
\hline $\begin{array}{l}\text { 4A. EXTRAVERSIÓN } \\
\text { 4B. INTROVERSIÓN }\end{array}$ & $\begin{array}{l}27,92 \\
10,05\end{array}$ & $\begin{array}{l}8,19 \\
7,26\end{array}$ & $\begin{array}{l}26,55 \\
10,81\end{array}$ & $\begin{array}{l}9,26 \\
7,17\end{array}$ & $\begin{array}{r}1,76 \\
-1,22\end{array}$ & $\begin{array}{l}0,079 \\
0,222\end{array}$ \\
\hline $\begin{array}{l}\text { 5A. SENSACIÓN } \\
\text { 5B. INTUICIÓN }\end{array}$ & $\begin{array}{l}17,92 \\
22,38\end{array}$ & $\begin{array}{l}6,43 \\
8,58\end{array}$ & $\begin{array}{l}18,64 \\
21,24\end{array}$ & $\begin{array}{l}6,01 \\
8,1\end{array}$ & $\begin{array}{r}-1,31 \\
1,55\end{array}$ & $\begin{array}{l}0,19 \\
0,122\end{array}$ \\
\hline $\begin{array}{l}\text { 6A. REFLEXIÓN } \\
\text { 6B. AFECTIVIDAD }\end{array}$ & $\begin{array}{l}13,34 \\
20,26\end{array}$ & $\begin{array}{l}7,6 \\
8,25\end{array}$ & $\begin{array}{l}18,39 \\
24,69\end{array}$ & $\begin{array}{l}8,05 \\
8,99\end{array}$ & $\begin{array}{r}-7,54 \\
7,54\end{array}$ & $\begin{array}{l}0,000^{*} \\
0,000^{*}\end{array}$ \\
\hline $\begin{array}{l}\text { 7A. SISTEMATIZACIÓN } \\
\text { 7B. INNOVACIÓN }\end{array}$ & $\begin{array}{l}35,01 \\
27,35\end{array}$ & $\begin{array}{r}11,04 \\
8,86 \\
\end{array}$ & $\begin{array}{l}36,91 \\
27,16 \\
\end{array}$ & $\begin{array}{c}11,41 \\
9\end{array}$ & $\begin{array}{r}-1,95 \\
0,25 \\
\end{array}$ & $\begin{array}{l}0,052^{\star} \\
0,804\end{array}$ \\
\hline
\end{tabular}


Tabla 5. Diferencias entre sexo en el grupo de españoles (Cont.)

\begin{tabular}{|c|c|c|c|c|c|c|}
\hline \multirow{2}{*}{$\frac{\text { Totales }}{\text { Escalas MIPS }}$} & \multicolumn{2}{|c|}{ España } & \multicolumn{2}{|c|}{ Argentina } & \multirow[b]{2}{*}{$\mathrm{T}$} & \multirow[b]{2}{*}{ N. Sig. } \\
\hline & Media & DT & Media & DT & & \\
\hline $\begin{array}{l}\text { 8A. RETRAIMIENTO } \\
\text { 8B. COMUNICATIVID. }\end{array}$ & $\begin{array}{l}16,19 \\
32,84\end{array}$ & $\begin{array}{r}8,68 \\
10,59\end{array}$ & $\begin{array}{l}18,15 \\
34,95\end{array}$ & $\begin{array}{l}9,56 \\
11,9\end{array}$ & $\begin{array}{l}-2,44 \\
-2,1\end{array}$ & $\begin{array}{l}0,015^{\star} \\
0,036^{\star}\end{array}$ \\
\hline $\begin{array}{l}\text { 9A. VACILACIÓN } \\
\text { 9B. FIRMEZA }\end{array}$ & $\begin{array}{l}17,76 \\
31,65\end{array}$ & $\begin{array}{l}10,7 \\
10,63\end{array}$ & $\begin{array}{l}14,84 \\
36,48\end{array}$ & $\begin{array}{l}10,43 \\
11,42\end{array}$ & $\begin{array}{r}3,16 \\
-5,09\end{array}$ & $\begin{array}{l}0,002^{*} \\
0,000^{*}\end{array}$ \\
\hline $\begin{array}{l}\text { 10A. DISCREPANCIA } \\
\text { 10B. CONFORMISMO }\end{array}$ & $\begin{array}{l}20,84 \\
16,88\end{array}$ & $\begin{array}{l}7,89 \\
4,2\end{array}$ & $\begin{array}{l}22,9 \\
16,45\end{array}$ & $\begin{array}{l}8,73 \\
4,81\end{array}$ & $\begin{array}{r}-2,79 \\
1,06\end{array}$ & $\begin{array}{l}0,006^{\star} \\
0,289\end{array}$ \\
\hline $\begin{array}{l}\text { 11A. SOMETIMIENTO } \\
\text { 11B. CONTROL }\end{array}$ & $\begin{array}{l}16,71 \\
23,56\end{array}$ & $\begin{array}{l}8,18 \\
7,02\end{array}$ & $\begin{array}{l}13,88 \\
27,98\end{array}$ & $\begin{array}{l}7,88 \\
8,07\end{array}$ & $\begin{array}{r}4,04 \\
-6,59\end{array}$ & $\begin{array}{l}0,000^{*} \\
0,000^{*}\end{array}$ \\
\hline $\begin{array}{l}\text { 12A. INSATISFACCIÓN } \\
\text { 12B. CONCORDANCIA }\end{array}$ & $\begin{array}{l}24,55 \\
33,8\end{array}$ & $\begin{array}{l}8,69 \\
8,14\end{array}$ & $\begin{array}{l}24,03 \\
27,93\end{array}$ & $\begin{array}{l}8,65 \\
8,61\end{array}$ & $\begin{array}{l}0,69 \\
8,15\end{array}$ & $\begin{array}{l}0,493 \\
0,000^{*}\end{array}$ \\
\hline $\begin{array}{l}\text { IMPRES. NEGATIVA } \\
\text { IMPRES. POSITIVA }\end{array}$ & $\begin{array}{l}3,31 \\
3,62\end{array}$ & $\begin{array}{l}2,42 \\
2,02\end{array}$ & $\begin{array}{l}2,58 \\
3,38\end{array}$ & $\begin{array}{l}2,2 \\
1,93\end{array}$ & $\begin{array}{l}3,73 \\
1,41\end{array}$ & $\begin{array}{l}0,000^{*} \\
0,16\end{array}$ \\
\hline
\end{tabular}

Nota: ${ }^{*} \mathrm{p}<0,05$

cientes de estructura se indican en la Tabla 6.

Tabla 6. Coeficientes de estructura de las escalas significativas en los datos del grupo de personas españolas díferenciadas por sexo

\begin{tabular}{llr}
\hline & & Función 1 \\
\hline S6B. & Afectividad & $\mathbf{0 , 7 1 5 0 1}$ \\
S12B. & Concordancia & $\mathbf{0 , 6 7 0 1 9}$ \\
S6A. & Reflexión & $-0,64964$ \\
S3A. & Individualismo & $-\mathbf{0 , 6 4 7 0 4}$ \\
S3B. & Protección & $\mathbf{0 , 6 4 6 5 5}$ \\
S11B. & Control & $-\mathbf{0 , 5 4 3 1 0}$ \\
S1B. & Preservación & $\mathbf{0 , 5 2 3 8 6}$ \\
S9B. & Firmeza & $-\mathbf{0 , 4 4 2 3 9}$ \\
S11A. & Sometimiento & $\mathbf{0 , 3 9 4 3 1}$ \\
S9A. & Vacilación & $\mathbf{0 , 3 8 7 3 7}$ \\
S1A. & Apertura & $-\mathbf{0 , 3 6 5 1 7}$ \\
S2B. & Acomodación & $\mathbf{0 , 3 4 2 2 7}$ \\
S10A. & Discrepancia & $-\mathbf{0 , 2 2 4 0 5}$ \\
S8B. & Comunicatividad & $-0,19607$ \\
S7A. & Sistematización & $-0,14601$ \\
SBA. & Retraimiento & $-0,08149$ \\
\hline
\end{tabular}

Por último, las diferencias de sexo en el grupo de personas argentinas se presenta en la Tabla 7. En esta tabla vemos que existen diferencias en 12 de las 24 escalas del MIPS.

Las variables que mejor discriminan entre sexo en el grupo de personas argentinas se obtuvieron mediante un análisis discriminante (función canónica: valor propio $=0,07$, varianza $=100 \%$, correlación canónica $=0,26$, lambda de Wilks $=$ 0,93, y $\chi^{2}(2)=21,37, p<0,0001$ ) cuyos coeficientes de estructura se presentan en la Tabla 8.

\section{DISCUSIÓN}

En el análisis de las diferencias entre personas españolas y argentinas se aprecia que los argentinos tienen puntuaciones promedio significativamente más altas en Apertura (tendencia a ver el lado bueno de las cosas, a ser más optimistas), Modificación (tendencia a autopercibirse como más activo) y Protección (disposición a satisfacer las necesidades de los demás), Reflexión (procesan los conocimientos por medio de la lógica y lo analítico), Afectividad (forman sus juicios respecto a sus propias reacciones afectivas), Firmeza (creen ser talentosos y competentes), Conformismo (respetuosos con la autoridad) y Concordancia (ocultan sus sentimientos negativos). Por el contrario los españoles tienen puntuaciones promedio más altas en Vacilación (tímidos y nerviosos en situaciones sociales), 
Tabla 7. Diferencias de sexo en el grupo de personas argentinas

\begin{tabular}{|c|c|c|c|c|c|c|}
\hline \multirow{2}{*}{$\begin{array}{c}\text { Totales } \\
\text { Escalas MIPS }\end{array}$} & \multicolumn{2}{|c|}{ España } & \multicolumn{2}{|c|}{ Argentina } & \multirow[b]{2}{*}{$T$} & \multirow[b]{2}{*}{ N. Sig. } \\
\hline & Media & DT & Media & DT & & \\
\hline $\begin{array}{l}\text { 1A. APERTURA } \\
\text { 1B. PRESERVACIÓN }\end{array}$ & $\begin{array}{l}25,2 \\
15,6\end{array}$ & $\begin{array}{l}7,1 \\
9,9\end{array}$ & $\begin{array}{l}26,2 \\
13,5\end{array}$ & $\begin{array}{l}6,5 \\
9,1\end{array}$ & $\begin{array}{r}-1,18 \\
1,87\end{array}$ & $\begin{array}{l}0,238 \\
0,062\end{array}$ \\
\hline $\begin{array}{l}\text { 2A. MODIFICACIÓN } \\
\text { 2B. ACOMODACIÓN }\end{array}$ & $\begin{array}{l}14,5 \\
18,2\end{array}$ & $\begin{array}{l}3,9 \\
9,0\end{array}$ & $\begin{array}{l}35,6 \\
16,2\end{array}$ & $\begin{array}{l}3,7 \\
9,1\end{array}$ & $\begin{array}{r}-3,01 \\
2,09\end{array}$ & $\begin{array}{l}0,003^{\star} \\
0,038^{*}\end{array}$ \\
\hline $\begin{array}{l}\text { 3A. INDIVIDUALISMO } \\
\text { 3B. PROTECCIÓN }\end{array}$ & $\begin{array}{l}18,1 \\
32,6\end{array}$ & $\begin{array}{l}7,3 \\
7,5\end{array}$ & $\begin{array}{l}20,4 \\
30,7\end{array}$ & $\begin{array}{l}6,9 \\
7,7\end{array}$ & $\begin{array}{r}-3,05 \\
2,17\end{array}$ & $\begin{array}{l}0,002^{*} \\
0,031^{*}\end{array}$ \\
\hline $\begin{array}{l}\text { 4A. EXTRAVERSIÓN } \\
\text { 4B. INTROVERSIÓN }\end{array}$ & $\begin{array}{r}28,1 \\
9,3\end{array}$ & $\begin{array}{l}8,2 \\
6,9\end{array}$ & $\begin{array}{l}28,2 \\
10,6\end{array}$ & $\begin{array}{l}8,0 \\
7,2\end{array}$ & $\begin{array}{l}-0,2 \\
-1,84\end{array}$ & $\begin{array}{l}0,841 \\
0,067\end{array}$ \\
\hline $\begin{array}{l}\text { 5A. SENSACIÓN } \\
\text { 5B. INTUICIÓN }\end{array}$ & $\begin{array}{l}17,2 \\
23,2\end{array}$ & $\begin{array}{l}6,5 \\
7,9\end{array}$ & $\begin{array}{l}18,6 \\
20,5\end{array}$ & $\begin{array}{l}6,2 \\
8,1\end{array}$ & $\begin{array}{r}-1,88 \\
2,82\end{array}$ & $\begin{array}{l}0,061 \\
0,005 *\end{array}$ \\
\hline $\begin{array}{l}\text { 6A. REFLEXIÓN } \\
\text { 6B. AFECTIVIDAD }\end{array}$ & $\begin{array}{l}12,4 \\
31,4\end{array}$ & $\begin{array}{l}6,9 \\
7,7\end{array}$ & $\begin{array}{l}16,2 \\
28,6\end{array}$ & $\begin{array}{l}6,8 \\
7,9\end{array}$ & $\begin{array}{r}-4,98 \\
3,05\end{array}$ & $\begin{array}{l}0,000^{\star} \\
0,002^{\star}\end{array}$ \\
\hline $\begin{array}{l}\text { 7A. SISTEMATIZACIÓN } \\
\text { 7B. INNOVACIÓN }\end{array}$ & $\begin{array}{l}36,2 \\
27,7\end{array}$ & $\begin{array}{r}11,1 \\
8,3\end{array}$ & $\begin{array}{l}38,7 \\
26,6\end{array}$ & $\begin{array}{r}11,2 \\
8,9\end{array}$ & $\begin{array}{r}-2,15 \\
0,87\end{array}$ & $\begin{array}{l}0,032^{\star} \\
0,385\end{array}$ \\
\hline $\begin{array}{l}\text { 8A. RETRAIMIENTO } \\
\text { 8B. COMUNICATIVID. }\end{array}$ & $\begin{array}{l}16,4 \\
33,9\end{array}$ & $\begin{array}{r}9,3 \\
10,3\end{array}$ & $\begin{array}{l}16,5 \\
36,2\end{array}$ & $\begin{array}{l}8,5 \\
9,7\end{array}$ & $\begin{array}{l}-0,09 \\
-2,16\end{array}$ & $\begin{array}{l}0,927 \\
0,031\end{array}$ \\
\hline $\begin{array}{l}\text { 9A. VACILACIÓN } \\
\text { 9B. FIRMEZA }\end{array}$ & $\begin{array}{l}15,9 \\
35,2\end{array}$ & $\begin{array}{r}10,3 \\
9,9\end{array}$ & $\begin{array}{l}14,3 \\
37,5\end{array}$ & $\begin{array}{l}9,0 \\
9,8\end{array}$ & $\begin{array}{r}1,57 \\
-2,25\end{array}$ & $\begin{array}{l}0,117 \\
0,025^{*}\end{array}$ \\
\hline $\begin{array}{l}\text { 10A. DISCREPANCIA } \\
\text { 10B. CONFORMISMO }\end{array}$ & $\begin{array}{l}19,3 \\
17,7\end{array}$ & $\begin{array}{l}8,3 \\
4,3\end{array}$ & $\begin{array}{l}19,2 \\
17,8\end{array}$ & $\begin{array}{l}7,6 \\
5,2\end{array}$ & $\begin{array}{l}-0,12 \\
-0,26\end{array}$ & $\begin{array}{l}0,906 \\
0,796\end{array}$ \\
\hline $\begin{array}{l}\text { 11A. SOMETIMIENTO } \\
\text { 11B. CONTROL }\end{array}$ & $\begin{array}{l}14,9 \\
24,7\end{array}$ & $\begin{array}{l}8,7 \\
6,7\end{array}$ & $\begin{array}{l}13,2 \\
26,5\end{array}$ & $\begin{array}{l}7,5 \\
6,8\end{array}$ & $\begin{array}{r}1,94 \\
-2,55\end{array}$ & $\begin{array}{l}0,053 \\
0,011^{\star}\end{array}$ \\
\hline $\begin{array}{l}\text { 12A. INSATISFACCIÓN } \\
\text { 12B. CONCORDANCIA }\end{array}$ & $\begin{array}{l}20,7 \\
34,2\end{array}$ & $\begin{array}{l}9,7 \\
7,3\end{array}$ & $\begin{array}{l}21,7 \\
31,3\end{array}$ & $\begin{array}{l}8,7 \\
7,8\end{array}$ & $\begin{array}{l}-1,1 \\
3,64\end{array}$ & $\begin{array}{l}0,27 \\
0,000^{\star}\end{array}$ \\
\hline $\begin{array}{l}\text { IMPRES.NEGATIVA } \\
\text { IMPRES.POSITIVA }\end{array}$ & $\begin{array}{l}3,3 \\
4,2\end{array}$ & $\begin{array}{l}2,3 \\
2,0\end{array}$ & $\begin{array}{l}2,9 \\
4,6\end{array}$ & $\begin{array}{l}2,2 \\
2,6\end{array}$ & $\begin{array}{r}1,87 \\
-1,55\end{array}$ & $\begin{array}{l}0,062 \\
0,122\end{array}$ \\
\hline
\end{tabular}

Nota: * $\mathrm{p}<0,05$

Tabla 8. Coeficientes de estructura de las escalas significativas en los datos del grupo de personas argentinas diferenciadas por sexo

\begin{tabular}{llc}
\hline & & Función 1 \\
\hline S6A. & Reflexión & $\mathbf{0 , 7 8 0 0 3}$ \\
S2A. & Modificación & 0,70284 \\
S7A. & Sistematización & 0,52523 \\
S9B. & Firmeza & $\mathbf{0 , 4 8 1 7 6}$ \\
S3A. & Individualismo & 0,48030 \\
S11B. & Control & 0,47250 \\
S6B. & Afectividad & $-0,40784$ \\
S8B. & Comunicatividad & 0,37391 \\
S12B. & Concordancia & $-0,34700$ \\
S2B. & Acomodación & $-0,31138$ \\
S5B. & Intuición & $-0,13915$ \\
S3B. & Protección & $-0,06858$ \\
\hline
\end{tabular}

Discrepancia (actúan de modo independiente y no conformista), Sometimiento (habituados al sufrimiento) e Insatisfacción (estados de ánimo y conductas variables).

El análisis discriminante nos aporta una función canónica significativa entre estos dos grupos de sujetos. Así, las dos variables que permiten diferenciar mejor entre los sujetos son, por este orden, Insatisfacción (estados de ánimo y conductas variables) y Protección (motivados a satisfacer primero a los demás). Este análisis permite clasificar adecuadamente al $68,79 \%$ de los participantes.

$\mathrm{Al}$ estudiar las diferencias entre mujeres españolas y argentinas observamos que las mujeres españolas tienen puntuaciones promedios más altas que las 
argentinas en las escalas Preservación (concentración en los problemas de la vida, agravándolos), Acomodación (no intentan dirigir sus vidas), Vacilación (tímidas y nerviosas en situaciones sociales), Discrepancia (actúan de modo independiente y no conformista), Sometimiento (habituados al sufrimiento) e Insatisfacción (estados de ánimo y conductas variables). Por el contrario las mujeres argentinas se autodescriben como más optimistas en cuanto al futuro, motivadas a satisfacer primero a los demás, creen ser talentosas y competentes y respetuosas con la autoridad.

Si comparamos hombres españoles y argentinos obtenemos que los primeros se autodescriben como más orientados a sus propias necesidades, procesan los conocimientos por medio de la lógica y lo analítico, actúan de modo independiente y no conformista y estados de ánimo y conductas variables. Los hombres argentinos intentan cambiar su entorno para conseguir sus deseos, motivados a satisfacer primero a los demás, forman sus juicios respecto a sus propias reacciones afectivas, respetuosos con la autoridad y ocultan sus sentimientos negativos.

Al analizar las diferencias de sexo en el grupo de participantes españoles se obtiene que las mujeres puntúan más alto en las escalas Preservación (concentración en los problemas de la vida, agravándolos), Acomodación (no intentan dirigir sus vidas), Protección (motivadas a satisfacer primero a los demás), Vacilación (tímidas y nerviosas en situaciones sociales), Sometimiento (habituadas al sufrimientos y sumisas ante los demás) y Concordancia (establecen vínculos afectivos y lealtades muy fuertes). Los hombres puntúan más alto que las mujeres en Apertura (optimistas en cuanto al futuro), Individualismo (orientados a sus propias necesidades sin preocuparse de los demás), Reflexión (procesan los conoci- mientos por medio de la lógica y lo analítico), Afectividad (se guían por sus valores personales para establecer juicios), Sistematización (transforman información nueva adecuándola a ìo conocido), Retraimiento (falta de emotividad e indiferencia social), Comunicatividad (buscan estimulación y atención), Firmeza (creen ser talentosos y competentes), Discrepancia (actúan de modo independiente y no conformista) y Control (ambiciosos y obstinados).

El análisis discriminante de las diferencias entre sexo de la muestra española clasifica adecuadamente al $68,17 \%$ de los participantes. En este análisis se incluyen 4 variables para la función que se presenta (aunque se presenten los datos de todas las variables consideradas), que son Preservación (concentración en los problemas de la vida, agravándolos), Protección (motivados a satisfacer primero a los demás), Reflexión (procesan los conocimientos por medio de la lógica y lo analítico) y Discrepancia (actúan de modo independiente y no conformista).

Las diferencias de sexo entre las personas argentinas denotan que los hombres argentinos se autodescriben intentando cambiar su entorno para conseguir sus deseos, orientados a sus propias necesidades sin preocuparse de los demás, procesan los conocimientos por medio de la lógica y lo analítico, organizados, buscan estimulación y atención, creen ser talentosos y competentes y son ambiciosos y obstinados. Las mujeres, por el contrario, se autodescriben no intentando dirigir sus vidas, prefieren lo simbólico y desconocido, forman sus juicios respecto a sus propias reacciones afectivas y ocultan sus sentimientos negativos.

El análisis discriminante nos aporta una función canónica entre hombres y mujeres significativa ( $p<0,0001$ ) clasificando al $59,74 \%$ de los participantes 
incluyendo sólo 2 variables de las 12 introducidas previamente. Estas variables son Reflexión (procesan los conocimientos por medio de la lógica y lo analítico) y Modificación (el futuro depende de ellos, por lo que intentan cambiar su entorno para conseguir sus deseos).

\section{CONCLUSIONES}

Consideramos que los datos obtenidos y analizados permiten enfatizar la importancia de ocuparse del estudio de las diferencias individuales contextualizadas. El estudio realizado es de tipo descriptivo exploratorio; invita a pensar que están en juego variables sociales, históricas y culturales que pueden dar cuenta, en el nivel explicativo, de las diferencias significativas encontradas.

Desde una perspectiva clínica, interesa destacar la necesidad de abordar la comprensión de los fenómenos patológicos tomando como línea base de análisis el conocimiento de los perfiles de la población general de pertenencia de los sujetos cuya conducta se quiere comprender, explicar y /o modificar. Además, la psicopatología está socioculturalmente estructurada y significada. Roles, normas, valores, expectativas, la deseabilidad social, las metas motivacionales, los estilos cognitivos así como las relaciones interpersonales que estructuran la deno- minada personalidad sana no son universalmente homogéneos (Casullo, 1996). Las diferencias encontradas según sexo pueden indicar cómo variables de tipo cultural estructuran comportamientos según género.

\section{REFERENCIAS BIBLIOGRÁFICAS}

Aparicio, M.E., y Sánchez-López, M.P. (en prensa). Los estilos de Personalidad: su medida a través del Inventario Millon de Estilos de Personalidad.

Casullo, M.M. (1996). Evaluación psicológica y psicodiagnóstico. Buenos Aires: Catálogos.

Millon, T. (1994). Millon Index of Personaliy Styles. Manual. San Antonio: The Psychological Corporation. (Traducción al español, Paidós, 1997).

Millon, T., Everly, G., y Davis, R.D. (1995). ¿Cómo puede facilitarse la integración de la psicoterapia mediante el conocimiento de la psicopatología? Una perspectiva a partir de los trastornos de la personalidad. Clínica y Salud, 6, 109-129.

Sánchez-López, M.P. (1997). El Estilo Psicológico como base del estudio de la diversidad humana: un ejemplo basado en los estilos de vida. Revista de Psicología, $X V$, 223-250.

Sánchez-López, M.P., y Aparicio, M.E. (1998). Inventario Millon de Estilos de Personalidad: su fiabilidad y validez en España y Argentina. Revista Iberoamericana de Diagnóstico y Evaluación Psicológica, 4, 87-110. 